\title{
Weighing graphene with QCM to monitor interfacial mass changes
}

Nurbek Kakenov, Osman Balci, Omer Salihoglu, Seung Hyun Hur, Sinan Balci, and Coskun Kocabas

Citation: Appl. Phys. Lett. 109, 053105 (2016); doi: 10.1063/1.4960299

View online: https://doi.org/10.1063/1.4960299

View Table of Contents: http://aip.scitation.org/toc/apl/109/5

Published by the American Institute of Physics

\section{Articles you may be interested in}

Controlling phase of microwaves with active graphene surfaces

Applied Physics Letters 110, 161102 (2017); 10.1063/1.4980087

Plasmon-polaritons on graphene-metal surface and their use in biosensors

Applied Physics Letters 100, 213110 (2012); 10.1063/1.4721453

Synthesis of graphene on gold

Applied Physics Letters 98, 183101 (2011); 10.1063/1.3584006

Mobility and saturation velocity in graphene on $\mathrm{SiO}_{2}$

Applied Physics Letters 97, 082112 (2010); 10.1063/1.3483130

Carbon dots decorated vertical $\mathrm{SnS}_{2}$ nanosheets for efficient photocatalytic oxygen evolution

Applied Physics Letters 109, 053905 (2016); 10.1063/1.4960527

Probing ultrafast energy transfer between excitons and plasmons in the ultrastrong coupling regime Applied Physics Letters 105, 051105 (2014); 10.1063/1.4892360

\section{PHYSICS TODAY}

MANAGER'S GUIDE WHITEPAPERS

\section{READ NOW}

PRESENTED BY

Accelerate R\&D with
Multiphysics Simulation 


\title{
Weighing graphene with QCM to monitor interfacial mass changes
}

\author{
Nurbek Kakenov, ${ }^{1}$ Osman Balci, ${ }^{1}$ Omer Salihoglu, ${ }^{1}$ Seung Hyun Hur, ${ }^{2}$ Sinan Balci, ${ }^{3}$ \\ and Coskun Kocabas ${ }^{1, a)}$ \\ ${ }^{1}$ Department of Physics, Bilkent University, 06800 Ankara, Turkey \\ ${ }^{2}$ School of Chemical Engineering, University of Ulsan, Daehak-ro 93, Nam-gu, Ulsan 680-749, South Korea \\ ${ }^{3}$ Department of Astronautical Engineering, University of Turkish Aeronautical Association, 06790 Ankara, \\ Turkey
}

(Received 20 May 2016; accepted 21 July 2016; published online 2 August 2016)

\begin{abstract}
In this Letter, we experimentally determined the mass density of graphene using quartz crystal microbalance (QCM) as a mechanical resonator. We developed a transfer printing technique to integrate large area single-layer graphene on QCM. By monitoring the resonant frequency of an oscillating quartz crystal loaded with graphene, we were able to measure the mass density of graphene as $\sim 118 \mathrm{ng} / \mathrm{cm}^{2}$, which is significantly larger than the ideal graphene $\left(\sim 76 \mathrm{ng} / \mathrm{cm}^{2}\right)$ mainly due to the presence of wrinkles and organic/inorganic residues on graphene sheets. High sensitivity of the quartz crystal resonator allowed us to determine the number of graphene layers in a particular sample. Additionally, we extended our technique to probe interfacial mass variation during adsorption of biomolecules on graphene surface and plasma-assisted oxidation of graphene. Published by AIP Publishing. [http://dx.doi.org/10.1063/1.4960299]
\end{abstract}

2-dimensional (2d) crystals provide a new platform to study physics in reduced dimensions. ${ }^{1}$ Graphene is at the center of this new field due to its unique physical properties. ${ }^{1}$ Various techniques have been implemented to elucidate the fundamental properties of graphene. For instance, optical spectroscopy has been demonstrated to measure the fine structure constant $(\alpha)$ defining visual transparency of graphene. ${ }^{2}$ Although the electrical and optical properties of graphene are extensively studied, the mechanical properties of graphene remain much less explored. Graphene yields tunable high mobility charge carriers for electronic and optoelectronic applications; on the other hand, graphene has also other physical properties such as monoatomic thickness together with strong covalent bonds, high stiffness, and low mass density. Due to its low mass density and extraordinary mechanical properties, graphene is an ideal nanomaterial for nanoelectromechanical systems (NEMS), e.g., for extremely small mass sensing. ${ }^{3,4}$ In fact, understanding basic properties of graphene plays a critical role in improving the performance of nano-electromechanical devices. Accurate measurements of these parameters are essential to model the performance of NEMS devices. For example, the elastic modulus of graphene has been measured to be $1 \mathrm{TPa}^{5} \mathrm{~A}$ variety of approaches used for the synthesis of graphene often produce single or polycrystalline graphene structures, thus leading to variations in the measured physical properties. ${ }^{6}$ For example, mechanical exfoliation yields single crystal graphene whereas chemical vapor deposition (CVD) most frequently yields polycrystalline graphene. Another parameter directly determining performance of NEMS devices is the mass density of graphene which is theoretically calculated to be $\sim 76 \mathrm{ng} / \mathrm{cm}^{2}{ }^{7,8}$ However, the mass density of CVD grown graphene has not yet been experimentally measured. In modeling of graphene NEMS devices, the value of $\sim 76 \mathrm{ng} / \mathrm{cm}^{2}$ is taken as the mass density of graphene ${ }^{7}$ but experimentally measured mass density of graphene

${ }^{a)}$ Electronic mail: ckocabas@fen.bilkent.edu.tr deviates from its theoretical value of $\sim 76 \mathrm{ng} / \mathrm{cm}^{2}$ since nanoscale wrinkles and (or) molecular adsorbates greatly affect the measured density. Herein, we used quartz crystal microbalance (QCM) to experimentally determine the mass density of graphene.

Integration of QCM with graphene yields a practical device for measuring the mass density and interfacial mass change of graphene. Various surface specific techniques have been implemented to understand these interfacial processes on graphene surface. ${ }^{9}$ Mass detection with mechanical resonators is an alternative method with qualities of low cost and extraordinary sensitivity, e.g., recently single nanoparticle weighing has been achieved. ${ }^{4,10}$ Graphene $^{7}$ and carbon nanotube ${ }^{11}$ based nano-electromechanical resonators have been demonstrated for extremely small mass detection. This configuration yields large quality factors of $10000-100000 .^{12,13}$ Owing to low cost and conceptual simplicity, quartz crystal microbalance provides sensitive means of in situ determination of interfacial mass change. ${ }^{14}$ QCM consists of a thin piezoelectric quartz crystal sandwiched between two metal electrodes. Application of an alternating voltage to the electrodes couples electric field to the mechanical oscillations resulting in a vibrational motion of the crystal at the resonance frequency. The quality factor of a QCM can exceed 100000 making QCM an ideal oscillator for sensing extremely small mass variations. ${ }^{13}$

We integrated QCM with graphene using a transferprinting process (Fig. 1). ${ }^{15}$ As a crystal, AT-cut, $\alpha$-quartz crystal with a mechanical response frequency of $5 \mathrm{MHz}$ was used. The exposed area of electrode is $\sim 1.37 \mathrm{~cm}^{2}$. The area of printed graphene is $0.64 \pm 0.05 \mathrm{~cm}^{2}$. Graphene samples were synthesized using CVD system. ${ }^{16}$ Graphene coated copper foils were spin coated with a photoresist (PR, AZ5214). A flat elastomeric stamp (PDMS) was placed on the PR layer and the copper foil was etched in $1 \mathrm{M}$ iron chloride. The stamp was applied to QCM and heated to $100^{\circ} \mathrm{C}$ in order to release graphene-PR layer. 

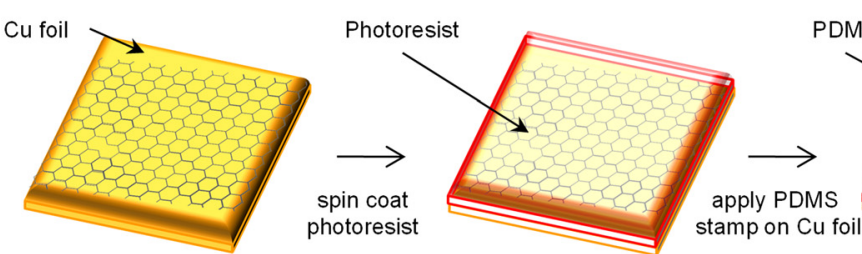

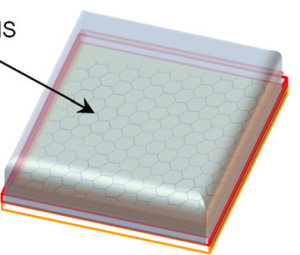

etch Cu foil

Graphene on $\mathrm{Cu}$

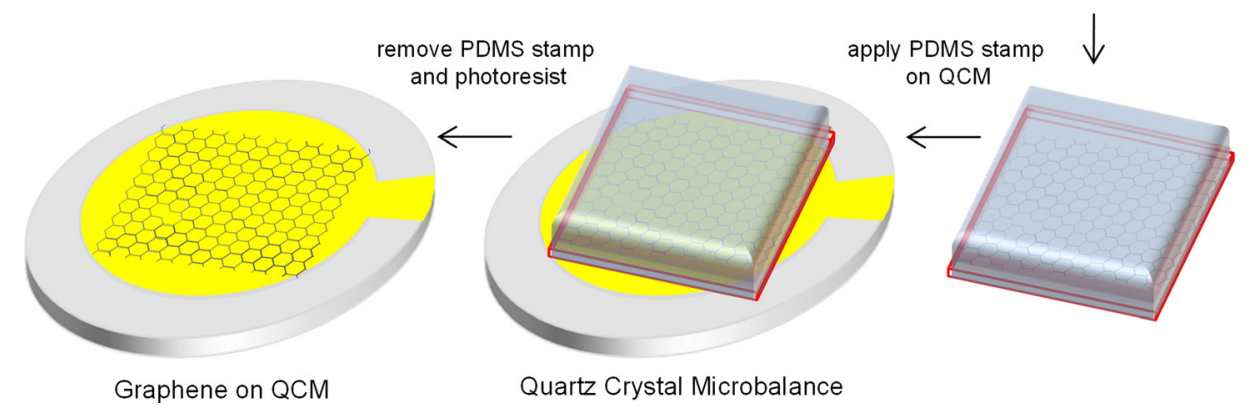

FIG. 1. Transfer printing process of graphene on the front electrode of a QCM.
Graphene coated QCM was mounted on a printedcircuit-board (PCB) (Fig. 2(a)). We fabricated a PCB and mounted the QCM on PCB. To verify graphene quality, we measured Raman scattering spectra of graphene sheets on copper substrate and on the gold electrode of QCM (Fig. 2(b)). ${ }^{17}$ The intensity ratio of $2 \mathrm{D} / \mathrm{G}$ is $\sim 3.4$ and the intensity of the defect mode (D) is negligible indicating high quality graphene on QCM.

To obtain resonance characteristics of QCM, we measured scattering parameters $\left(\mathrm{S}_{11}\right)$ using a two-port vector network analyzer (VNA, HP 8753D) (Fig. 2(c)). S-parameters elucidate the electromechanical properties of QCM and the interaction with the surrounding medium. One port of the VNA is connected to QCM mounted on a crystal holder with a BNC connector. We measured S-parameters for a frequency range of $1 \mathrm{kHz}$ at $5 \mathrm{MHz}$ where the magnitude of $S_{11}$

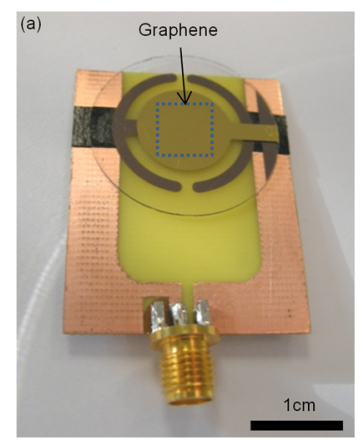

(c)

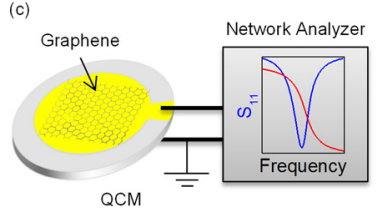

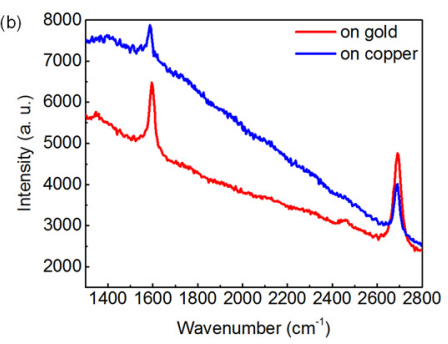
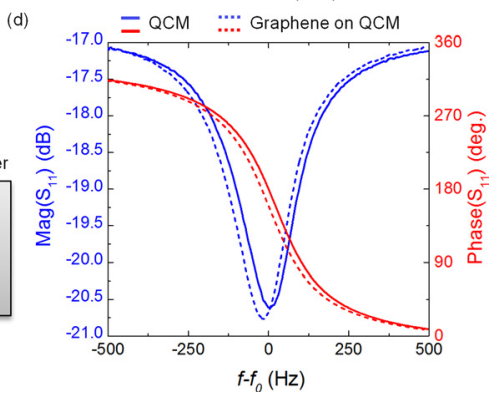

FIG. 2. (a) A photograph of a QCM mounted on a printed circuit board. (b) Raman scattering spectra of graphene on gold and on copper films. (c) Experimental setup used for probing resonance characteristic of the QCM. (d) Magnitude and phase of measured scattering parameter $\mathrm{S}_{11}$ of port-1, as a function of frequency for blank (solid line) and graphene coated QCM (dot line). The resonance frequency is $5007323.4 \mathrm{~Hz}$. The Q-factor of the resonator is $\sim 22600$. After coating the surface of QCM with $0.64 \mathrm{~cm}^{2}$ graphene, we observed $\sim 20 \mathrm{~Hz}$ shift in the frequency. reaches a minimum value since the total impedance attains its minimum value (Fig. 2(d)). Next, we transferred a single layer graphene and measured $\mathrm{S}_{11}$ (Fig. 2(d)). The area of graphene is $0.64 \mathrm{~cm}^{2}$, which is smaller than the area of the front electrode $1.37 \mathrm{~cm}^{2}$. After graphene transfer, the resonance frequency decreases by $20.5 \mathrm{~Hz}$.

Additionally, we integrated QCM with multilayer graphene and measured scattering parameters (Figs. 3(a) and 3(b)). We found that the resonance frequency linearly increases with an increase in number of graphene layers. The scattered plot in Fig. 3(c) demonstrates the variation of the resonance frequency with the layer number. The red line shows a
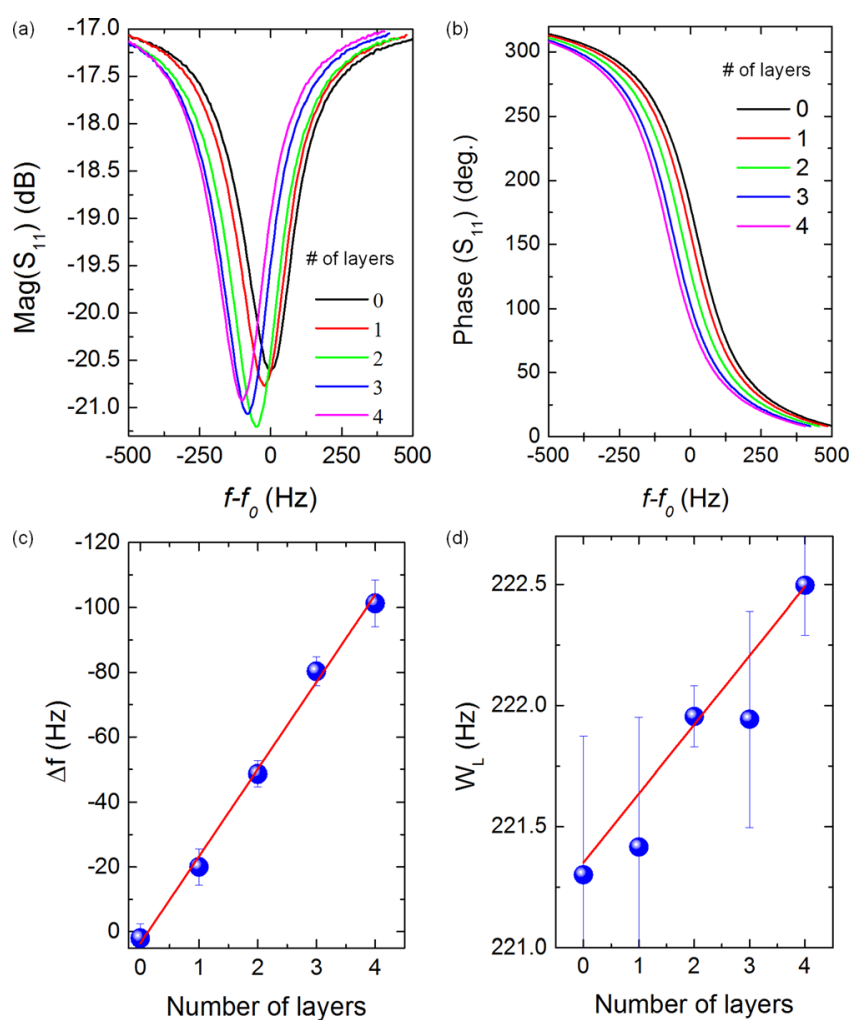

FIG. 3. Magnitude (a) and phase (b) of scattering parameters measured for QCM with multilayer graphene. (c) Measured frequency shift as a function of graphene layer numbers. (d) The bandwidth of the QCM vs. number of graphene layers. 

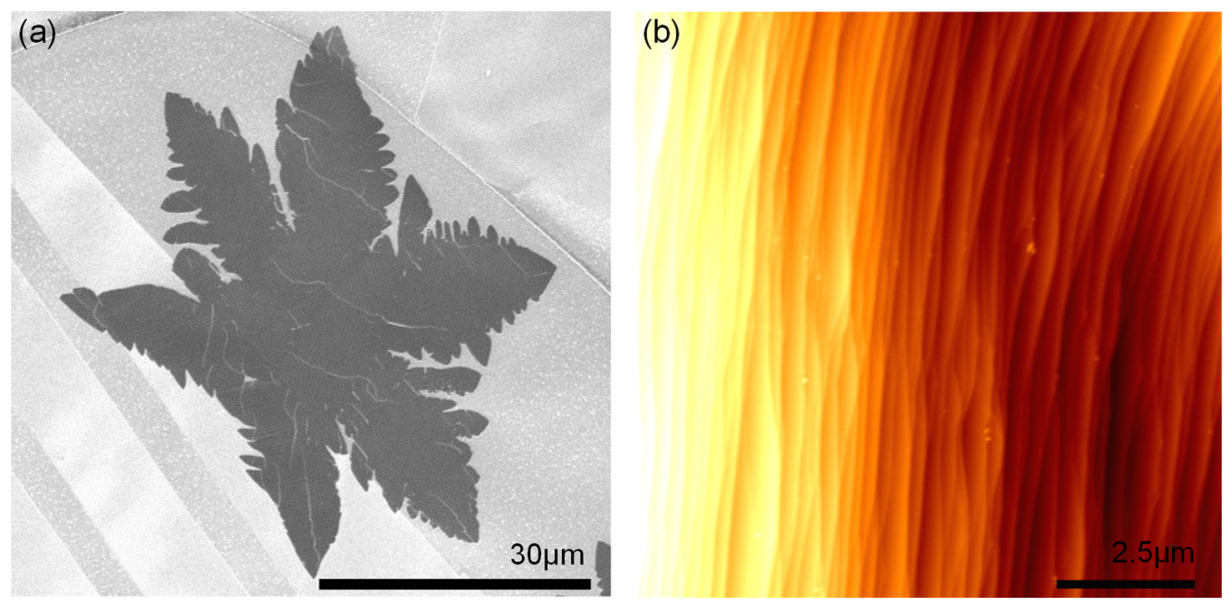

FIG. 4. (a) Scanning electron microscope and (b) atomic force microscope images of wrinkled graphene flakes on copper foils. Obviously, nanometersized wrinkles are formed on graphene.

curve obtained by linear least squares fitting. The slope of the fitting curve provides the interfacial mass change of $26 \pm$ $2 \mathrm{~Hz}$ for each graphene layer. Figure 3(d) shows the bandwidth of the QCM as a function of graphene layers.

We then quantified the density of graphene from the measured frequency shifts. The resonance frequency of the crystal is sensitive to variation in interfacial mass as summarized in Sauerbrey's equation ${ }^{18}$

$$
\Delta f=-\frac{2 n f_{0}^{2}}{\sqrt{\rho_{q} \mu_{q}}} \Delta m,
$$

where $f_{0}$ is the resonant frequency of quartz crystal, and $n$ is the number of the harmonic at which the crystal is driven. For a 5-MHz, AT cut quartz crystal, the mass density is $\rho_{q}=2.648 \mathrm{~g} \mathrm{~cm}^{-3}$, and the shear modulus is $\mu_{\mathrm{q}}=2.947 \times 10^{11} \mathrm{~g} \mathrm{~cm}^{-1} \mathrm{~s}^{-2}$. Here, $\Delta m$ defines the change in mass. Sauerbrey's equation, however, provides an average mass density, and in most cases, necessitates calibration. To quantify the frequency change and mass uptake, we calibrated the QCM by a known amount of mass. A gold film with a thickness of $20 \mathrm{~nm}$ was evaporated on QCM. ${ }^{19}$ We measured a shift of $4.80 \mathrm{kHz}$ in resonant frequency. The density of the evaporated gold thin film is $16 \mathrm{~g} / \mathrm{cm}^{3}$ corresponding to the mass uptake of $20.5 \mu \mathrm{g} .{ }^{20}$ For an exposed area of $0.64 \mathrm{~cm}^{2}$, the calibration factor is $4.27 \mathrm{ng} / \mathrm{Hz}$. For each graphene layer, we obtained $26 \mathrm{~Hz}$ frequency shift corresponding to a mass uptake of $111 \mathrm{ng}$. It should be noted here that this value includes both the mass of graphene and organic/inorganic residues. The extent of the residual contamination on graphene has been characterized by employing a variety of techniques. ${ }^{21}$ Besides, we performed additional experiments to determine the amount of chemical residues on the QCM. To quantify the mass of residues, we performed a similar transfer process without growing graphene on copper foils. The observed frequency shift for each transfer process is $8.3 \mathrm{~Hz}$ corresponding to a mass uptake of $35 \mathrm{ng}$. After subtracting the residue mass from the measured mass and normalizing by the area, we estimated the mass density of graphene as $\sim 118 \mathrm{ng} / \mathrm{cm}^{2}$. This value is significantly larger than the theoretical value of $\sim 76 \mathrm{ng} / \mathrm{cm}^{2}$ calculated from the mass density of bulk graphite. ${ }^{7}$ The large mass density of graphene is most likely due to the wrinkles formed on graphene as shown in
Figs. 4(a) and 4(b). ${ }^{22}$ The wrinkles increase the surface area and mass density of graphene. Previously, high densities of wrinkles were observed in large-scale grown graphene on metallic substrates. ${ }^{22}$ These wrinkles are formed due to thermal contraction of the substrate during the cooling process. Besides, nanoscale holes, cracks, and organic/ inorganic residues alter the measured mass density of graphene. There are also other sources of errors originating from the calibration procedure, e.g., the thickness and mass density of gold film used for the calibration and also uncertainties in the measured area of graphene.

We now describe application of graphene modified QCM mass sensor in biomolecule detection (Fig. 5(a)). Recently, single nanoparticle mass and position have been simultaneously measured using a nanomechanical resonator. ${ }^{10} \mathrm{By}$ integrating the QCM sensor (Stanford Research, QCM200) with a flow chamber, we monitored the binding dynamics of proteins on graphene. ${ }^{23}$ Quartz crystal is placed

(a)

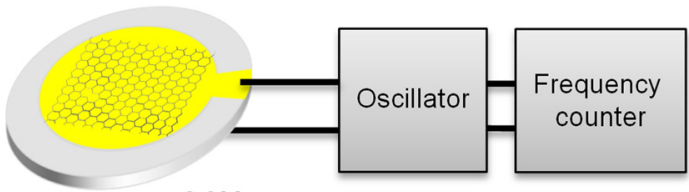
QCM

(b)

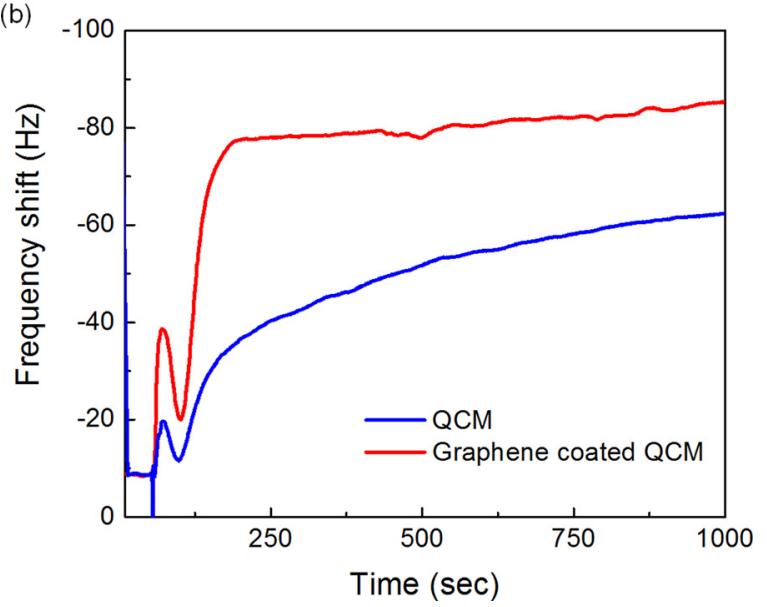

FIG. 5. (a) Schematics of the experimental setup used to probe time trace of the resonance frequency of the QCM. (b) Overlaid time trace of resonance frequency indicating binding kinetics of BSA $(100 \mathrm{nM})$ on bare and graphene coated QCM surfaces. 

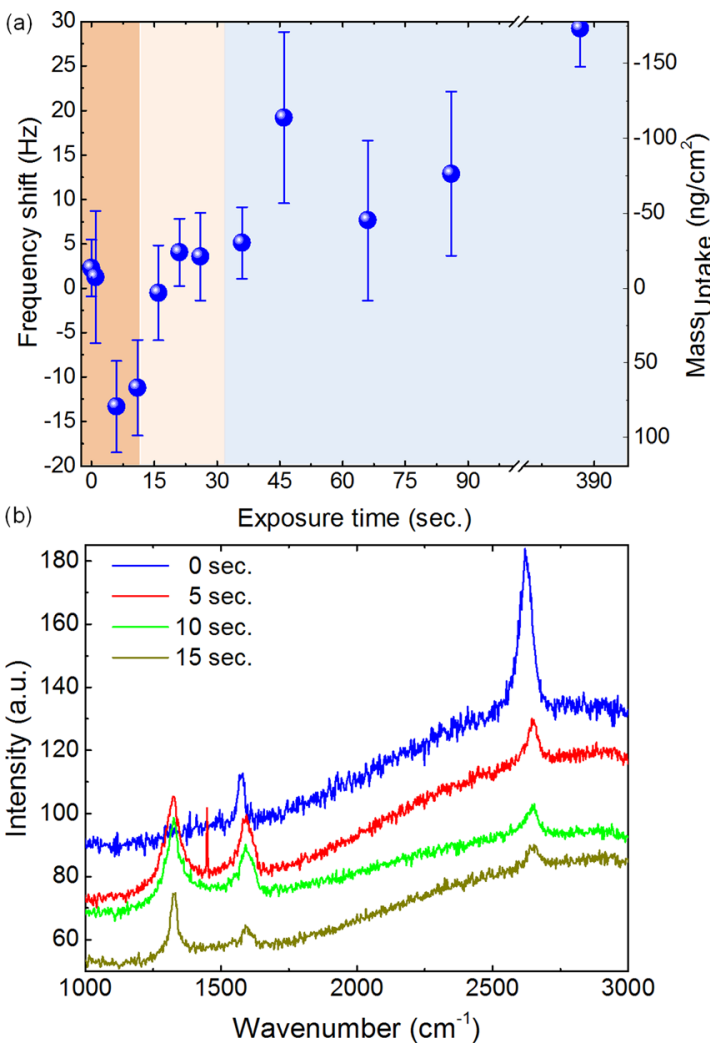

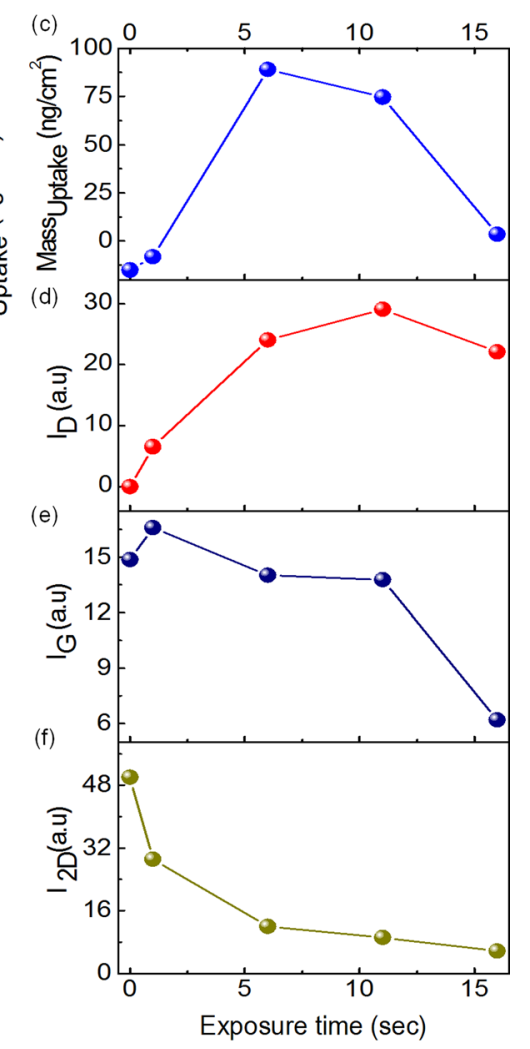

FIG. 6. Probing oxidation of graphene by mass detection. (a) Variation of the resonance frequency and associated mass uptake of graphene exposed to oxygen plasma. (b) Raman spectra of graphene with various exposure times. (c)-(f) Mass uptake of graphene and correlated Raman intensity for D, G, and $2 \mathrm{D}$ bands. in a feedback loop of an oscillator. The time trace of the resonance frequency was monitored by means of a frequency counter. The kinetic parameters of the nonspecific binding on a surface can be extracted from the time trace of the resonance frequency (Fig. 5(b)). We used $100 \mathrm{nM}$ of Bovine serum albumin (BSA) protein to study nonspecific binding on bare and graphene coated gold electrodes. The time constants, obtained by fitting the time trace data, are $390 \mathrm{~s}$ and $30 \mathrm{~s}$, for bare and graphene modified QCMs, respectively. From the measured time constants, we estimated the association constants of BSA on bare and graphene coated gold surfaces as $0.26 \mathrm{M}^{-1} \mathrm{~s}^{-1}$ and $3.3 \mathrm{M}^{-1} \mathrm{~s}^{-1}$, respectively.

In addition, sensitive mass detection enabled by QCM can be used for monitoring chemical reactions on graphene. Therefore, we traced oxidation of graphene by monitoring the interfacial mass change and correlating the mass change with Raman spectroscopy. QCM with graphene was exposed to oxygen plasma (Fig. 6). It is well known that graphene undergoes chemical oxidation when exposed to plasma. ${ }^{24} \mathrm{We}$ directly correlate the mass uptake with the structure of graphene using Raman spectrum of graphene after each oxygen plasma exposure (Fig. 6(b)). In the diagram in Fig. 6(a), we observe three different regimes. In $10 \mathrm{~s}$ exposure, interfacial mass increases by $93 \mathrm{ng} / \mathrm{cm}^{2}$ (Fig. 6(c)). This mass uptake is most likely due to the oxidation of graphene and adsorption of other molecules from the plasma. The intensity increase in the D-band $\left(1950 \mathrm{~cm}^{-1}\right)$ confirms the formation of $\mathrm{sp}$ bonds, associated with lattice distortions. The decrease in intensity of 2D-band $\left(2700 \mathrm{~cm}^{-1}\right)$ indicates deformation of graphene. After $15 \mathrm{~s}$ exposure, a drastic decrease was observed in the interfacial mass, which is most likely due to $\mathrm{CO}_{2}$ release that is expected for the early stage of graphene oxidation. When we further increased the exposure time, we observed complete removal of graphene. This step could include physical removing of small graphene/graphene oxide flakes due to bombarding of surface by energetic oxygen molecules. Correlating the interfacial mass change with Raman spectra could provide more useful information for understanding interfacial reactions on graphene (Figs. 6(e) and 6(f)).

In conclusion, owing to its low cost and ease of use, QCM sensor is a practical way to study the interfacial processes on graphene. By integrating QCM with graphene, we were able to experimentally determine the mass density of graphene. The discrepancy between the theoretical and experimental values is mainly due to (i) organic/inorganic residues, (ii) wrinkles, and (iii) water molecules on graphene. In addition, we traced the adsorption of biomolecules on graphene modified QCM in real-time. The hydrophobicity of graphene could be the main dominant effect that enhances the associativity of proteins by more than 12 times. Besides, chemical reactions on the oxygen plasma treated graphene have been monitored. Indeed, this method can be further developed to investigate interfacial mechanisms on graphene.

This work was supported by the Scientific and Technological Research Council of Turkey (TUBITAK) Grant No. 113F278. N.K. acknowledges the fellowship from TUBITAK-BIDEB 2215 PhD Fellowship Programme. C.K. also acknowledges the support from the European Research Council (ERC) Consolidator Grant No. ERC - 682723 SmartGraphene.

${ }^{1}$ K. S. Novoselov, A. K. Geim, S. V. Morozov, D. Jiang, Y. Zhang, S. V. Dubonos, I. V. Grigorieva, and A. A. Firsov, Science 306(5696), 666 (2004).

${ }^{2}$ R. R. Nair, P. Blake, A. N. Grigorenko, K. S. Novoselov, T. J. Booth, T. Stauber, N. M. R. Peres, and A. K. Geim, Science 320(5881), 1308 (2008). 
${ }^{3}$ J. S. Bunch, A. M. van der Zande, S. S. Verbridge, I. W. Frank, D. M. Tanenbaum, J. M. Parpia, H. G. Craighead, and P. L. McEuen, Science 315(5811), 490 (2007).

${ }^{4}$ Y. T. Yang, C. Callegari, X. L. Feng, K. L. Ekinci, and M. L. Roukes, Nano Lett. 6(4), 583 (2006)

${ }^{5}$ C. Lee, X. D. Wei, J. W. Kysar, and J. Hone, Science 321(5887), 385 (2008).

${ }^{6}$ L. Lin, J. Y. Li, H. Y. Ren, A. L. Koh, N. Kang, H. L. Peng, H. Q. Xu, and Z. F. Liu, ACS Nano 10(2), 2922 (2016).

${ }^{7}$ C. Y. Chen, S. Rosenblatt, K. I. Bolotin, W. Kalb, P. Kim, I. Kymissis, H. L. Stormer, T. F. Heinz, and J. Hone, Nat. Nanotechnol. 4(12), 861 (2009).

${ }^{8}$ T. Fang, A. Konar, H. L. Xing, and D. Jena, Phys. Rev. B 84(12), 125450 (2011).

${ }^{9}$ Y. Q. Bie, J. Horng, Z. W. Shi, L. Ju, Q. Zhou, A. Zettl, D. P. Yu, and F. Wang, Nat. Commun. 6, 7593 (2015).

${ }^{10}$ S. Olcum, N. Cermak, S. C. Wasserman, and S. R. Manalis, Nat. Commun. 6, 7070 (2015).

${ }^{11}$ H. Y. Chiu, P. Hung, H. W. C. Postma, and M. Bockrath, Nano Lett. 8(12), 4342 (2008).

${ }^{12}$ M. D. Ward and D. A. Buttry, Science 251(4999), 1372 (1991); D. A. Buttry and M. D. Ward, Chem. Rev. 92(6), 1355 (1992); V. M. Mecea, Sens. Actuators, A 128(2), 270 (2006); V. M. Mecea, Anal. Lett. 38(5), 753 (2005); V. M. Mecea, J. Therm. Anal. Calorim. 86(1), 9 (2006).
${ }^{13}$ M. D. Ward and D. A. Buttry, Science 249(4972), 1000 (1990).

${ }^{14}$ C. Mak and J. Krim, Phys. Rev. B 58(9), 5157 (1998); J. Y. Park and M. Salmeron, Chem. Rev. 114(1), 677 (2014).

${ }^{15}$ O. Salihoglu, S. Balci, and C. Kocabas, Appl. Phys. Lett. 100(21), 213110 (2012).

${ }^{16}$ X. S. Li, W. W. Cai, J. H. An, S. Kim, J. Nah, D. X. Yang, R. Piner, A. Velamakanni, I. Jung, E. Tutuc, S. K. Banerjee, L. Colombo, and R. S. Ruoff, Science 324(5932), 1312 (2009).

${ }^{17}$ A. C. Ferrari, J. C. Meyer, V. Scardaci, C. Casiraghi, M. Lazzeri, F. Mauri, S. Piscanec, D. Jiang, K. S. Novoselov, S. Roth, and A. K. Geim, Phys. Rev. Lett. 97(18), 187401 (2006).

${ }^{18}$ G. Sauerbrey, Z. Phys. 155(2), 206 (1959).

${ }^{19}$ See supplementary material at http://dx.doi.org/10.1063/1.4960299 for the optical microscopy images of graphene on $\mathrm{SiO}_{2} / \mathrm{Si}$ surface and atomic force microscopy image of $20 \mathrm{~nm}$ gold film on QCM.

${ }^{20}$ J. Siegel, O. Lyutakov, V. Rybka, Z. Kolska, and V. Svorcik, Nanoscale Res. Lett. 6, 96 (2011).

${ }^{21}$ Y. B. Zhou, R. O'Connell, P. Maguire, and H. Z. Zhang, Sci. Rep. 4, 7032 (2014); Y. C. Lin, C. C. Lu, C. H. Yeh, C. H. Jin, K. Suenaga, and P. W. Chiu, Nano Lett. 12(1), 414 (2012).

${ }^{22}$ W. J. Zhu, T. Low, V. Perebeinos, A. A. Bol, Y. Zhu, H. G. Yan, J. Tersoff, and P. Avouris, Nano Lett. 12(7), 3431 (2012).

${ }^{23}$ K. K. Kanazawa and J. G. Gordon, Anal. Chem. 57(8), 1770 (1985).

${ }^{24}$ B. Ozyilmaz, P. Jarillo-Herrero, D. Efetov, and P. Kim, Appl. Phys. Lett. 91(19), 192107 (2007). 